BROOKHAVEN NATIONAL LABORATORY

Associated Universities, Inc.

Upton, New York

AGS DIVISION TECHNICAT NOTE

NO. 110

\title{
K. Prelec
}

June 4,1974

STRIPPING OF $\mathrm{H}^{-}$IN A MAGNETTC FIELD

Stripping of $\mathrm{H}^{-}$into $\mathrm{H}^{\circ}$ in a magnetic field has been considered as a possible mode of charge exchange $\mathrm{H}^{-}$injection into a storage ring (1); it would be followed by a second stripping $\mathrm{H}^{\circ} \rightarrow \mathrm{H}^{+}$in the ring itself. The main advantage of this mode compared to the standard mode $\mathrm{H}^{-} \rightarrow \mathrm{H}^{+}$is the straight path of $\mathrm{H}^{\mathrm{O}}$ particles through ring magnets, between the magnetic stripper and the ring stripper. In this note a possible application to the charge exchange injection into the AGS will be discussed.

When a charged particle moves in a magnetic field, the equivalent electric field in the rest frame of the particle is given by

$E=\gamma v B=c \beta \gamma B$

Decay of $\mathrm{H}^{-}$ions in a magnetic field may be represented by the differential equation

$$
\frac{d f(t)}{d t}=-\frac{f(t)}{\tilde{c}(t)}
$$

In this expression $f(t)$ is the fraction of the initial $\mathrm{H}^{-}$beam and $\widetilde{c}(t)$ the mean life time of an ion in the magnetic field. In a homogeneous magnetic field $\widetilde{c}(t)$ is a constant. It has been found ${ }^{(2)}$ that the expression

$$
\widetilde{c}(E)=\frac{C}{E} \exp \left(\frac{D}{E}\right)
$$

fits we11 the experimental data, with constants 


$$
\begin{aligned}
& \mathrm{C}=7.96 \times 10^{-6} \mathrm{Vs} / \mathrm{m} \\
& D=4.256 \times 10^{9} \mathrm{~V} / \mathrm{m} .
\end{aligned}
$$

Stripping in a homogeneous magnetic field is less attractive because mean life times would be too long and angular dispersion of resulting neutral particles too large (in order to avoid any appreciable stripping in the fringing field the main field cannot be arbitrarily high, with the consequence that $\tilde{c}$ becomes relatively long). A better alternative is to use a very strong magnetic field and to try to accomplish the stripping in the steep fringing field. The simplest approximation for the fringing field is

$$
B=B_{0} \frac{x}{d}=B_{0} y \quad 0 \leq y \leq 1
$$

where $d$ is the assumed axial length of that field, and often taken as to be equal to the gap width. For such a field

$$
\widetilde{c}=\frac{C}{c \beta \gamma \beta \beta_{o} y} \exp \left(\frac{D}{c \beta \gamma B}\right)=\frac{C_{1}}{y} \exp \left(\frac{D_{1}}{y}\right) \text {. }
$$

After changing variables from $t$ to $y$ we have

$$
\frac{d f}{d t}=\frac{d f}{d y} \frac{d y}{d t}=\frac{\beta c}{d} \frac{d f}{d y}
$$

Substituting for $\widetilde{c}$ and separating the variables we get

$$
\frac{d f}{f}=-\frac{d}{\beta c} \frac{d y}{c}=-\frac{d}{c_{1} \beta c} y \exp \left(-\frac{1}{y}\right) d y
$$

Integration yields

$$
\begin{aligned}
& \ln f=-\frac{d}{C_{1} \beta c} \int_{0}^{y} y \exp \left(-\frac{D_{1}}{y}\right) d y \\
& f=\exp \left[-\frac{d}{C_{1} \beta c} \int_{0}^{y} y \exp \left(-\frac{D_{1}}{y^{2}}\right) d y\right] .
\end{aligned}
$$


The multiplicative integration constant is equal to 1 because $f(y=0)=1$. This integral has to be evaluated by a numerical method. The distribution of neutral particles ofwil be

$$
f_{0}=1-f
$$

For the purpose of injection the angular dispersion of neutral particles has to be estimated. Before a $\mathrm{H}^{-}$ion has been dissociated, its trajectory will be deflected by an angle

$$
\Theta=\frac{B_{0} d}{2(B \rho)} y^{2}
$$

where $\mathrm{y}$ is the path length from the edge of the fringing field to the point where the dissociation takes place. It will, however, be more important, to calculate the radial distribution of neutral particles at the position of the ring stripper (conversion of $\mathrm{H}^{\circ}$ into $\mathrm{H}^{+}$). If $\mathrm{I}$ is the distance between the two strippers, and $l$ the deflection at the ring stripper from the position corresponding to $\Theta=0$, then

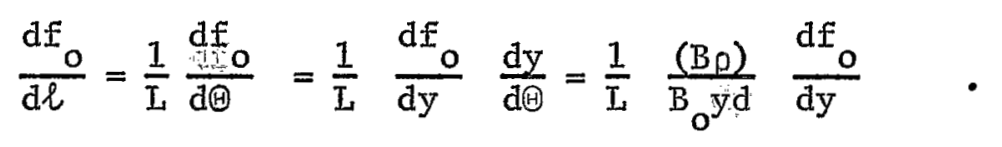

Substitution

$$
\frac{d f_{o}}{d y}=\frac{d}{\beta c} \frac{f(y)}{c(y)}
$$

results in

$$
\frac{\mathrm{df} o}{\mathrm{dl}}=\frac{1}{\mathrm{I}} \frac{(\mathrm{B} \rho)}{\mathrm{B}_{\mathrm{o}} \beta \mathrm{c}} \quad \frac{1}{\mathrm{y}} \frac{\mathrm{f}(\mathrm{y})}{\tilde{c}(\mathrm{y})}
$$

which is a function of $\frac{\ell}{L}$ via

$$
\frac{l}{\mathrm{~L}}=\Theta=\frac{\mathrm{B}_{\mathrm{o}} \mathrm{d}}{2(\mathrm{BP})} \mathrm{y}^{2} \text {. }
$$


An example has been worked out for the AGS injection, assuming

$$
\begin{aligned}
& \mathrm{B}_{\mathrm{o}}=40 \mathrm{kG} \quad(=4 \mathrm{~T}) \\
& \mathrm{d}=5 \mathrm{~cm} .
\end{aligned}
$$

Fig. 1 shows the distribution function $I \frac{d f_{o}}{d l}$ vs. $\frac{l}{I}$. It is clear that even if the magnetic stripper was only $\mathrm{L}=10 \mathrm{~m}$ from the ring stripper, the radial distribution of neutral particles would be too wide.

\section{References}

1. Design Study for a Medium - Energy, Intense, Proton-Storage Ring (WNR Ring), LA - 4946.

2. G. M. Stinson, et a1., Nuc1. Instrum. Methods, 74, 333 (1964). 
